\title{
On integro-differential equations of parabolic and elliptic type
}

\author{
by H. UGowski (Gdańsk)
}

Krzyżański ([5], [6]) considered linear integro-differential equations of parabolic type and showed that the weak maximum principle known for a linear parabolic partial differential equation can be extended to the integro-differential equation. As a consequence he obtained a uniqueness theorem for solutions of the first Fourier problem for the integrodifferential equation in an unbounded region. Some more general theorems concerning a system of non-linear integro-differential inequalities of parabolic type were proved by Łojczyk-Królikiewicz and Szarski ([8], [9]). Similar results for parabolic differential inequalities containing functionals can be found in reference [10].

In this paper we are dealing with the existence of solutions of the first initial-boundary value problem for the following system of parabolic integro-differential equations:

$$
\sum_{i, j=1}^{n} a_{i j}^{k}(x, t) u_{x_{i} x_{j}}^{k}+\sum_{i=1}^{n} b_{i}^{k}(x, t) u_{x_{i}}^{k}+c^{k}(x, t) u^{k}-u_{t}^{k}
$$

$$
\begin{array}{r}
=f^{k}\left(x, t, u^{1}, \ldots, u^{N}, u_{x_{1}}^{1}, \ldots, u_{x_{u}}^{1}, \ldots, u_{x_{1}}^{N}, \ldots, u_{x_{n}}^{N} \int_{G_{t}} u^{1}(y, t) \mu^{1}(x, t ; d y), \ldots\right. \\
\left.\ldots, \int_{G_{t}} u^{N}(y, t) \mu^{N}(x, t ; d y)\right), \quad k=1, \ldots, N .
\end{array}
$$

We take advantage of well-known theorems on existence and Schauder estimates of solutions of a single linear parabolic equation.

At first we consider a system more general than (0.1) containing some operators $B^{k} u$ on the right-hand side. For bounded regions wo formulate two existence theorems. The proof of Theorem 1 is based on Schauder's fixed point theorem and is patterned on the proof given by A. Friedman for a single semilinear parabolic partial differential equation ([4], p. 204). Theorem 2 is proved by means of the successive approximations method, making use of the Banach fixed point theorem. The latter 
method, ensuring uniqueness as well, is also employed to solve the Cauchy problem for the system in question. The theorems mentioned above involve as a particular case system (0.1).

Some of the results obtained for parabolic equations are carried over to elliptic equations in section 6.

$I$ wish to express my best thanks to $P$. Besala for suggesting the problem and giving me valuable hints.

1. Preliminary lemmas for the parabolic case in bounded domains. Let $G$ be a bounded open domain of the Euclidean space $E_{n+1}$ of the variables $(x, t)=\left(x_{1}, \ldots, x_{n}, t\right)$, enclosed by two domains $R_{0}$ and $R_{r}$ lying on the planes $t=0$ and $t=T=$ const $>0$ respectively, and by a manifold $S$ situated in the strip $0<t \leqslant T$. The parabolic distance of points $P(x, t), P^{\prime}\left(x^{\prime}, t^{\prime}\right) \in E_{n+1}$ is defined as

$$
d\left(P, P^{\prime}\right)=\left(\left|x-x^{\prime}{ }_{i}^{2}+\right| t-t^{\prime} \mid\right)^{1 / 2}, \quad \text { where }\left|x-x^{\prime}\right|=\left[\sum_{i=1}^{n}\left(x_{i}-x_{i}^{\prime}\right)^{2}\right]^{1 / 2} .
$$

We shall make use of the definition of Hölder continuity with exponent $a, 0<a<1$, included in [4].

Let us introduce the following norms:

$$
\begin{aligned}
& |u|_{0}^{\theta}=\sup _{P \in G^{\prime}}|u(P)|, \quad|u|_{a}^{a}=|u|_{0}^{G}+\sup _{P, P^{\prime} \in G^{\prime}} \frac{\left|u(P)-u\left(P^{\prime}\right)\right|}{\left[d\left(P, P^{\prime}\right)\right]^{\alpha}}, \\
& |u|_{1+a}^{G}=|u|_{a}^{G+}+\sum_{i=1}^{n}\left|u_{x_{i}}\right|_{a}^{(i}, \\
& |u|_{2+a}^{G}=|u|_{a}^{G}+\sum_{i=1}^{n}\left|u_{x_{i}}\right|_{a}^{G}+\sum_{i, j=1}^{n}\left|u_{x_{i} x_{j}}\right|_{a}^{G}+\left|u_{l}\right|_{a}^{G} \quad(0<a<1) .
\end{aligned}
$$

Denote by $C_{k+a}(G)(k=0,1,2)$ the set of all functions $u$ for which $|u|_{k+a}^{\theta}<\infty$. The following norms will also be needed:

$$
|u|_{1-0}^{G}=|u|_{0}^{G}+\sup _{P, P^{\prime} \in G} \frac{\left|u(P)-u\left(P^{\prime}\right)\right|}{\left|x-x^{\prime}\right|+\left|t-t^{\prime}\right|}, \quad|u|_{2-0}^{G}=|u|_{1-0}^{G}+\sum_{i=1}^{G}\left|u_{x_{i}}\right|_{1-0}^{G} .
$$

The set of all functions $u$ for which $|u|_{k-0}^{G}<\infty \quad(k=1,2)$ will be denoted by $C_{k-0}(\theta)$.

Suppose that for every point $P$ of the closure $\bar{S}$ there exists an $(n+1)$-dimensional neighbourhood $V$ such that $V \cap \bar{S}$ can be represented, for some $i(1 \leqslant i \leqslant n)$, by an equation of the form

$$
x_{i}=h\left(x_{1}, \ldots, x_{i-1}, x_{i+1}, \ldots, x_{n}, t\right) .
$$


If the functions $h$ belong to $C_{\omega}(\omega=a, 1+a, 2+\alpha, 1-0,2-0)$, then we say that $S$ is of class $C_{w}$. If $S \in C_{2+a}$ and the derivatives $h_{x t}$ exist and are continuous, then we say that $S$ is of class $\bar{C}_{2+a}$; if, moreover, $h_{t}$ exist and are continuous, then $S$ is said to belong to class $\overline{\bar{C}}_{2+a}$. For the manifold $S$ of class $C_{\omega}$ there exist a finite number of balls $V^{k}$ covering $\bar{S}$ such that $S^{k}=\bar{S} \cap V^{k}$ can be represented by the equation

$$
x_{i_{k}}=h^{k}\left(x_{1}, \ldots, x_{i_{k-1}}, x_{i_{k}+1}, \ldots, x_{n}, t\right),
$$

where $h^{k} \in C_{\omega}$.

Let $v(x, t)$ be a function defined on the manifold $S$ of class $C_{w}$. Using (1.1) we can write the function $v(x, t)$ on $S^{k}$ as a function of variables $\left(x_{1}, \ldots, x_{i_{k}-1}, x_{i_{k}+1}, \ldots, x_{n}, t\right)$ in a certain region $S_{0}^{k}$. We then define

$$
|v|_{\omega}^{\mathbf{S}}=\max _{k}|v|_{\omega}^{S_{0}^{k}}
$$

and we say that $v \in C_{\omega}(S)$ if $|v|_{\omega}^{S}<\infty$.

A function $\varphi(x, t)$ defined on the parabolic boundary $\Sigma=\bar{R}_{0} \cup S$ is said to be of class $C_{2+a}(G)$ if there exists a function $\Phi \in C_{2+\sigma}(G)$ such that $\Phi=\varphi$ on $\Sigma$. We then define $|\varphi|_{2+a}^{a}=\inf _{\Phi}|\Phi|_{2+\alpha}^{a}$. If $S \in C_{2+a}$, then for any extension $\Phi$ of $\varphi$, the derivatives $\Phi_{x_{l}}, \Phi_{x_{x} x}, \Phi_{l}$ are uniquely defined (by continuity) on the boundary $\partial R_{0}$ of domain $R_{0}$, and the definition is independent of $\Phi$. We denote these derivatives (on $\partial R_{0}$ ) by $\varphi_{x_{t}}, \varphi_{x_{i} x_{x}}, \varphi_{t}{ }^{\left({ }^{1}\right)}$.

Let us consider the first initial-boundary value problem for the linear parabolic equation:

$$
\begin{gathered}
L u=\sum_{i, j=1}^{n} a_{i j}(x, t) u_{x_{i} x_{j}}+\sum_{i=1}^{n} b_{t}(x, t) u_{x_{i}}+c(x, t) u-u_{t}=f(x, t), \\
(x, t) \epsilon \bar{G} \backslash \Sigma, \\
u(x, t)=\varphi(x, t), \quad(x, t) \epsilon \Sigma .
\end{gathered}
$$

By a solution of equation (1.2) we shall always understand a regular solution, i.e. continuous in the domain $\bar{G}$ and possessing in $\bar{G} \backslash \Sigma$ continuous derivatives appearing in $L u$.

The following assumptions will be needed:

(A) For any $(x, t) \epsilon \bar{G}$ and $\xi \in E_{n}$ we have $a_{i j}(x, t)=a_{j i}(x, t)$,

$$
\sum_{i, j=1}^{n} a_{i j}(x, t) \xi_{i} \xi_{j} \geqslant K_{0}|\xi|^{2} \quad\left(K_{0}>0\right) .
$$

(B) The coefficients of $L$ and the function $f(x, t)$ are uniformly Hölder continuous (exponent $\alpha$ ) in $\bar{G}$.

(2) All the definitions stated above are taken from [4] (pp. 61.65, 190). 
Then there exists a constant $K_{1}>0$ such that

$$
\left|a_{i j}\right|_{a}^{G}, \quad\left|b_{i}\right|_{a}^{G}, \quad|c|_{a}^{G} \leqslant K_{1} .
$$

(C) The coefficients $a_{i j}$ are uniformly Hölder continuous (exponent $a$ ) in $\vec{G}$ and belong to $C_{1-0}(S) ; b_{i}$ and $c$ are continuous in $\bar{G}$.

Thus for some constants $K_{2}, K_{3} \geqslant 0$

$$
\sum_{i, j=1}^{n}\left|a_{i j}\right|_{\alpha}^{\alpha}+\sum_{i=1}^{n}\left|b_{i}\right|_{0}^{G}+|c|_{0}^{G} \leqslant K_{2}, \quad \sum_{i, j=1}^{n}\left|a_{i j}\right|_{1-0}^{S} \leqslant K_{3} .
$$

Now we state two lenmas included in [4].

LEMMA 1 ([4], p. 65). Let assumptions (A) and (B) hold true. If $\varphi \in C_{2+a}, S \in \overline{\bar{C}}_{2+a}$ and $L \varphi=f$ on $\partial R_{0}$, then there exists a unique solution $u$ of problem (1.2), (1.3), and furthermore $u \in C_{2+a}(G)$. Moreover, there exists a constant $K$ depending only on $K_{0}, K_{1}, \alpha$ and domain $G$ such that

$$
|u|_{2+a}^{G} \leqslant K\left(|\varphi|_{2+a}^{G}+|f|_{a}^{G}\right) .
$$

Lemma 2 ([4], p. 191). Assume that $S \in C_{2+a} \cap C_{2-0}$ and that (A), (C) hold true. Let $f(x, t)$ be a continuous function in $\bar{G}$ vanishing on $a R_{0}$ and let $u(x, t)$ be a solution of the problem

$$
L u=f(x, t) \quad \text { in } \bar{G} \backslash \Sigma, \quad u=0 \quad \text { on } \Sigma
$$

Then for any $\beta, 0<\beta<1$, there exists a constant $K^{\prime}$ depending only on $\beta, K_{0}, K_{2}, K_{3}$ and $G$ such that

$$
|u|_{1+\beta}^{G} \leqslant K^{\prime}|f|_{0}^{G} .
$$

Remark. From the proof of this lemma follows the existence of a constant $\vec{K}$ depending on the same parameters as $K^{\prime}$ and such that

$$
|u|_{1+\beta}^{\theta^{\tau}} \leqslant \bar{K} \tau^{(1-\beta) / 2}|f|_{0}^{\theta^{\tau}}, \quad \text { where } G^{\tau}=G \frown\{(x, t): t<\tau\}, 0<\tau \leqslant T \text {. }
$$

Now we shall prove some lemmas on functions defined by an integral. Put $G_{t}=\{x:(x, t) \in \bar{G} \backslash \bar{S}\}$ and denote by $m$ the $n$-dimensional Lebesgue measure.

LEMma 3. If the manifold $S$ is of class $C_{2+a}$, then there exists a constant $x$ depending only on $S$ and such that

$$
m\left(G_{i} \backslash G_{t^{\prime}}\right) \leqslant *\left|t-t^{\prime}\right|
$$

Proof. Denote by $W^{k}$ any $(n+1)$-dimensional cube

$$
\left\{\varepsilon_{k} \leqslant x_{i} \leqslant \delta_{k}(i=1, \ldots, n), \bar{\varepsilon}_{k} \leqslant t \leqslant \bar{\delta}_{k}\right\} \quad\left(\delta_{k}-\varepsilon_{k}=\bar{\delta}_{k}-\bar{\varepsilon}_{k}, \bar{\varepsilon}_{k} \geqslant 0\right) .
$$


The assumption of the lemma implies the existence of a finite number of cubes $W^{k}\left(k=1, \ldots, k_{1}\right)$ with centres on $\bar{S}$, covering $\bar{S}$ and such that every manifold $S^{k}=W^{k} \cap \vec{S}$ can be represented by (1.1), where $h^{k} \in C_{2+a}$. Hence, writing

$$
S_{\pi \tau^{\prime}}^{k}=S^{k} \cap\left\{(x, t): \tau \leqslant t \leqslant \tau^{\prime}\right\} \quad\left(\tau<\tau^{\prime}\right)
$$

and using the formula for the surface area, we immediately obtain

$$
m\left(\boldsymbol{S}_{\tau \tau^{\prime}}^{k}\right) \leqslant\left(\tau^{\prime}-\tau\right)\left(\delta_{k}-\varepsilon_{k}\right)^{n-1} \cdot \sup \left[1+\sum_{\substack{i=1 \\ i \neq i_{k}}}^{n}\left(h_{x_{k}}^{k}\right)^{2}+\left(h_{t}^{k}\right)^{2}\right]^{1 / 2}=\varkappa_{k}\left(\tau^{\prime}-\tau\right) .
$$

It is easy to see that the set $\left(G_{\tau} \cup G_{\tau^{\prime}}\right) \backslash\left(G_{\tau} \cap G_{\tau^{\prime}}\right)$ is contained in the projection of the manifold

$$
S_{\tau \tau^{\prime}}=S \cap\left\{(x, t): \tau \leqslant t \leqslant \tau^{\prime}\right\}
$$

on the plane $t=0$. Since the $n$-dimensional Lebesgue measure of any $n$-dimensional manifold is not less than that of the projection of the manifold on a plane of the same dimension, therefore by (1.6) we have

$$
\begin{aligned}
m\left(\left[G_{\tau} \cup G_{\tau^{\prime}}\right] \backslash\left[G_{\tau} \cap G_{\tau^{\prime}}\right]\right) & \leqslant m\left(S_{\tau \tau^{\prime}}\right) \leqslant m\left(\sum_{k=1}^{k_{2}} S_{\tau \tau^{\prime}}^{k}\right) \leqslant \sum_{k=1}^{k_{1}} m\left(S_{\tau \tau^{\prime}}^{k}\right) \\
& \leqslant\left(\tau^{\prime}-\tau\right) \sum_{k=1}^{k_{1}} \varkappa_{k}=\varkappa\left(\tau^{\prime}-\tau\right)
\end{aligned}
$$

and the lemma follows.

Denote by the $\sigma$-field of all Lebesgue-measurable subsets of the domain

$$
D_{0}=\overline{0 \leqslant t \leqslant T^{\prime}} G_{t} .
$$

We shall make use of a non-negative measure $\mu(x, t ; D)$ (depending on $(x, t) \epsilon \bar{G})$ defined on $\mathcal{K}$ which satisfies the following conditions:

(1) there is a constant $M_{1}>0$ such that for any $(x, t) \in \bar{G}$

$$
\mu\left(x, t ; D_{0}\right) \leqslant M_{1}
$$

(2) there exists a finite non-negative measure $\bar{\mu}$ defined on th such that for any $D \in \mathcal{M}$ and any points $P(x, t), P^{\prime}\left(x^{\prime}, t^{\prime}\right)$ of the domain $\bar{G}$ we have

$$
\left|\mu(x, t ; D)-\mu\left(x^{\prime}, t^{\prime} ; D\right)\right| \leqslant \bar{\mu}(D)\left[d\left(P, P^{\prime}\right)\right]^{\gamma},
$$

where $0<\gamma<1$ is a constant.

If $G$ is not a cylindrical domain (i.e. a domain which cannot be represented as the topological product of a domain in $E_{n}$ by an interval of variable $t$ ), we additionally assume that 
(3) for any $D \in \mathcal{M}$ there is a positive constant $M_{2}$ such that

$$
\mu(x, t ; D) \leqslant M_{2} m(D),
$$

$m(D)$ being the Lebesgue measure of $D$.

As a simple example of a measure satisfying conditions (1) and (2) may serve a measure given by the formula

$$
\mu(x, t ; D)=\varrho(x, t) \bar{\mu}(D), \quad(x, t) \in \bar{G}, D \in \mathcal{H},
$$

$\varrho(x, t)$ being a function non-negative and uniformly Hölder continuous in $\bar{G}$.

LEMMA 4. We assume that the measure $\mu$ satisfies conditions (1) and (2), or, if $G$ is not a cylindrical domain, we assume that $\mu$ satisfies conditions (1), (2) and (3) and that $S \in C_{2+a}$. Moreover, suppose that $u(x, t)$ is uniformly Hölder continuous with exponent $a$ in $\bar{G}$.

Then the function

$$
v(x, t)=\int_{\theta_{t}} u(y, t) \mu(x, t ; d y)
$$

is uniformly Hölder continuous with exponent $\delta=\min (\alpha, \gamma)$ in $\bar{G}$.

Proof. Since $u \in C_{\mathrm{a}}(G)$, the integral

$$
\int_{G_{t}} u(y, t) \mu(x, t ; d y)
$$

exists. Making use of the known properties of integrals, we obtain

$$
v(x, t)-v\left(x^{\prime}, t^{\prime}\right)=I_{1}+I_{2}-I_{3}+I_{4}-I_{5},
$$

where

$$
\begin{gathered}
I_{1}=\int_{\sigma_{t} \cap G_{t^{\prime}}}\left[u(y, t)-u\left(y, t^{\prime}\right)\right] \mu(x, t ; d y), \quad I_{2}=\int_{a_{t} \cap a_{t^{\prime}}} u\left(y, t^{\prime}\right) \mu(x, t ; d y), \\
I_{3}=\int_{\sigma_{t} \cap G_{t^{\prime}}} u\left(y, t^{\prime}\right) \mu\left(x^{\prime}, t^{\prime} ; d y\right), \quad I_{4}=\int_{G_{t} \backslash G_{t^{\prime}}} u(y, t) \mu(x, t ; d y), \\
I_{5}=\int_{G_{t^{\prime}} \backslash G_{t}} u\left(y, t^{\prime}\right) \mu\left(x^{\prime}, t^{\prime} ; d y\right)\left({ }^{2}\right) .
\end{gathered}
$$

Further, we have

$$
\begin{aligned}
\left|I_{1}\right| & \leqslant \int_{G_{t} \cap G_{t^{\prime}}}\left|u(y, t)-u\left(y, t^{\prime}\right)\right| \mu(x, t ; d y) \leqslant M_{3}\left|t-t^{\prime}\right|^{a^{\prime 2}} \int_{G_{t} \cap G_{t^{\prime}}} \mu(x, t ; d y) \\
& =M_{3}\left|t-t^{\prime}\right|^{a / 2} \mu\left(x, t ; G_{t} \cap G_{t^{\prime}}\right) \leqslant M_{1} M_{3} T^{(a-\delta) / 2}\left[d\left(P, P^{\prime}\right)\right]^{d}
\end{aligned}
$$

where $P=(x, t), P^{\prime}=\left(x^{\prime}, t^{\prime}\right), M_{3}=\sup _{P, P^{\prime} \in G} \frac{\left|u(P)-u\left(P^{\prime}\right)\right|}{\left[d\left(P, P^{\prime}\right)\right]^{a}}$.

(9) If $G$ is a cylindrical domain, then for any $t, t^{\prime} \in[0, T]$ we have $G_{\imath}=G_{t^{\prime}}=D_{0}$ and consequently $I_{4}=I_{5}=0$, 
Direct application of the definition of integral and condition (2) yield the estimate

$$
\left|I_{2}-I_{3}\right| \leqslant \bar{\mu}\left(G_{l} \cap G_{t^{\prime}}\right) \sup |u|\left[d\left(P, P^{\prime}\right)\right]^{\gamma} \leqslant \bar{\mu}\left(D_{0}\right)|u|_{0}^{\sigma}\left[d\left(P, P^{\prime}\right)\right]^{\gamma} .
$$

If $G_{t} \backslash G_{l^{\prime}} \neq O$, then it follows from Lemma 3 and from condition (3) (3) that

$$
\left|I_{4}\right| \leqslant M_{2}|u|_{0}^{\sigma} \varkappa\left|t-t^{\prime}\right| \leqslant M_{2}|u|_{0}^{\sigma} \varkappa T^{1-\delta / 2}\left[d\left(P, P^{\prime}\right)\right]^{\delta}
$$

and the same inequality is true for $I_{5}$. The estimates obtained and (1.7) imply the inequality

$$
\begin{aligned}
\mid v(x, t) & -v\left(x^{\prime}, t^{\prime}\right) \mid \\
& \leqslant\left(M_{1} M_{3} T^{(a-\delta) / 2}+\bar{\mu}\left(D_{0}\right)|u|_{0}^{G} R^{\gamma-\delta}+2 M_{2}|u|_{0}^{G} T^{1-\delta / 2}\right)\left[d\left(P, P^{\prime}\right)\right]^{\delta},
\end{aligned}
$$

$R$ being the diamater in the parabolic distance of domain $G$. This completes the proof.

2. Existence theorems for parabolic equations in bounded domains. Set $u(x, t)=\left(u^{1}(x, t), \ldots, u^{N}(x, t)\right), \bar{u}(x, t)=\left(\overline{u^{1}}(x, t), \ldots, \overline{u^{N}}(x, t)\right)$, where $u^{k}(x, t), \overline{u^{k}}(x, t) \in C_{1+\varepsilon}(G)$. We define the following operations and norm:

$$
u+\bar{u}=\left(u^{1}+\overline{u^{1}}, \ldots, u^{N}+\overline{u^{N}}\right), \quad \eta u=\left(\eta u^{1}, \ldots, \eta u^{N}\right)
$$

( $\eta$ is a real number),

$$
|u|_{l+\varepsilon}^{G}=\sum_{k=1}^{N}\left|u^{k}\right|_{l+\varepsilon}^{G} .
$$

Then the set $C_{l+\varepsilon}^{N}(G)$ of all vector-functions $u(x, t)$ for which $|u|_{l+\varepsilon}^{G}<\infty$ is a Banach space.

For every $0<\tau \leqslant T$ let $B^{k}(k=1, \ldots, N)$ be an operator defined on the set of all vector-functions $u=\left\{u^{k}\right\}$ regular in $\overline{G^{\tau}}$ with values belonging to the set of all functions defined in $\overline{G^{\tau}} \backslash \Sigma^{\tau}$, where $G^{\tau}=G \cap$ $\cap\{(x, t): 0<t<\tau\}$ and $\Sigma^{\tau}=\Sigma \cap\{(x, t): 0<t \leqslant \tau\}$.

We shall consider the first boundary problem

$$
\begin{gathered}
L^{k} u^{k} \equiv \sum_{i, j=1}^{n} a_{i j}^{k}(x, t) u_{x_{i x j}}^{k}+\sum_{i=1}^{n} b_{i}^{k}(x, t) u_{x_{i}}^{k}+c^{k}(x, t) u^{k}-u_{t}^{k}=B^{k} u, \\
(x, t) \in \overline{G^{\tau} \backslash} \backslash \Sigma^{\tau} \\
u^{k}(x, t)=\phi^{k}(x, t), \quad(x, t) \in \Sigma^{\tau} \quad(k=1, \ldots, N) .
\end{gathered}
$$

(3) If $G$ is a cylindrical domain, then assumption $S^{*} \in C_{2+a}$ and condition (3) are superfluous. 
The following assumptions are introduced $(i, j=1, \ldots, n ; k=1, \ldots$ $\ldots, N ; 0<\tau \leqslant T)$ :

I. For any $(x, t) \in \bar{G}$ and $\xi \in E_{n}$ we have $a_{i j}^{k}(x, t)=a_{j i}^{k}(x, t)$, $\sum_{i, j=1}^{n} a_{i j}^{k}(x, t) \xi_{i} \xi_{j} \geqslant K_{0}|\xi|^{2}$ ( $K_{0}$ is a positive constant).

II. The coefficients of $L^{k}$ satisfy the uniform Hölder condition with exponent $a$ in $\bar{G}$ and, moreover, $a_{i j}^{k} \in C_{1-0}(S)$.

III. The manifold $S$ belongs both to $\overline{\bar{C}}_{2+a}$ and to $C_{2 \cdots 0}$.

IV. The functions $\varphi^{k}$ are of class $C_{1+\beta} \cap C_{2+\alpha}(\alpha<\beta<1)$.

V. If $\Phi \in C_{1+\beta}^{N}\left(G^{\tau}\right) \frown C_{2+a}^{N}\left(G^{\tau}\right)$ and $\Phi=\varphi$ on $\Sigma^{\pi}$, then $B^{k} \Phi=L^{k} \varphi^{k}$ on $\partial R_{0}$.

VI. Operators $B^{k}$ map the space $C_{1+a}^{N}\left(G^{\tau}\right)$ into the set $\underset{0<\varepsilon<1}{\bigcup} C_{\varepsilon}\left(G^{\boldsymbol{\tau}}\right)$ and there are constants $A_{1}, A_{2}, A_{3} \geqslant 0,0 \leqslant \lambda<1$ such that for any $u \in C_{2+a}^{N}\left(G^{\tau}\right)$ the following inequality holds:

$$
\left|B^{k} u\right|_{0}^{G^{\tau}} \leqslant A_{1}+A_{2}\left(|u|_{1}^{\sigma^{\pi}}\right)^{\lambda}+A_{3}|u|_{1}^{G^{\tau}}
$$

where

$$
|u|_{1}^{\sigma^{\tau}}=\left.\sum_{k=1}^{N}\left|u^{\left.k_{1}\right|_{0}}+\sum_{k=1}^{N} \sum_{i=1}^{n}\right| u_{x_{i}}^{k}\right|_{0} ^{\sigma^{\tau}}
$$

VII. Operators $B^{k}$ are continuous in the space $C_{1+\alpha}^{N}\left(G^{\tau}\right)$; more precisely, if $u, u_{l} \in C_{1+a}^{N}\left(G^{\tau}\right)$ and $\lim _{l \rightarrow \infty}\left|u_{l}-u\right|_{1+u}^{G^{\tau}}=0$, then $\lim _{l \rightarrow \infty}\left|B^{k} u_{l}-B^{k} u\right|_{0}^{G^{\tau}}=0$.

By assumption II inequalities (1.4) and (1.5) hold true for coefficients of $L^{k}(k=1, \ldots, N)$.

Let $F^{k}(x, t)$ be continuous functions in $G^{\tau}$ and let $L^{k} \varphi^{k}=F^{k}$ $(k=1, \ldots, N)$ on $\partial R_{0}$. Then it follows from assumptions I-IV and from the remark to Lemma 2 that there is a constant $\bar{K}(\theta)(\theta=\alpha, \beta)$ depending only on $\theta, K_{0}, K_{2}, K_{3}$ and domain $G$ such that for any solution $u^{k}(x, t)$ $(k=1, \ldots, N)$ of the problem

$$
L^{k} u^{k}=F^{k} \quad \text { in } \overline{G^{\natural}} \backslash \Sigma^{\gamma}, \quad u^{k}=\varphi^{k} \quad \text { on } \Sigma^{n}
$$

we have

$$
\left|u^{k}\right|_{1+\theta}^{\sigma^{\tau}} \leqslant \bar{K}(\theta) \tau^{(1-0) / 2}\left(\left|F^{k}\right|_{0}^{\sigma^{\tau}}+\left|L^{k} \Phi^{k}\right|_{0}^{\sigma^{\tau}}\right)+\left|\Phi_{\mid}^{k}\right|_{1+\theta}^{\sigma^{\boldsymbol{r}}}
$$

where $\Phi^{k} \in C_{1+\beta}\left(G^{\tau}\right) \frown C_{2+a}\left(G^{\tau}\right)$ is any extension of $\varphi^{k}$.

THEOREM 1. If assumptions I-VII are satisfied and

$$
\bar{K}(\alpha) N A_{3} \tau^{(1-\alpha) / 2}<1,
$$

then there exists a solution $u(x, t)=\left\{u^{k}(x, t)\right\}$ of problem (2.1), (2.2); furthermore, $u \in C_{1+\beta}^{N}\left(G^{\tau}\right) \cap C_{2+\varepsilon}\left(G^{\tau}\right)$ for some $\varepsilon, 0<\varepsilon<1$. 
Proof. Denote by $C_{M}$ the set of all functions $u(x, t) \in C_{1+a}^{N}\left(G^{z}\right)$ such that $|u|_{1+a}^{\sigma^{x}} \leqslant M$ and $u(x, t)=\varphi(x, t)$ on $\Sigma^{\tau}$, where the constant $M>0$ will be specified later. Now for $u \in C_{M}$ consider the problem

$$
\begin{gathered}
L^{k} v^{k}=B^{k} u \equiv F^{k}(x, t), \quad(x, t) \in \overline{G^{\natural}} \Sigma^{\tau}, \\
v^{k}(x, t)=\varphi^{k}(x, t), \quad(x, t) \in \Sigma^{\tau} \quad(k=1, \ldots, N) .
\end{gathered}
$$

By virtue of assumptions I-VI and Lemma 1, problem (2.7), (2.8) possesses a unique solution $v(x, t)$ which belongs to $C_{2+\varepsilon}^{N}\left(G^{\tau}\right)$ for some $0<\varepsilon<1$. Moreover, by $(2.5), v(x, t)$ belongs to $C_{1+\beta}^{N}\left(G^{\tau}\right)$.

Now we define on $C_{M}$ a transformation $Z$ setting $v=Z u$. Using Schauder's theorem, we shall prove that $Z$ has a fixed point. We first show that $Z$ maps $C_{M}$ into itself.

In view of (2.3) we have

$$
\left|F^{k}\right|_{0}^{G^{\tau}} \leqslant A_{1}+A_{2} M^{\lambda}+A_{3} M \quad(k=1, \ldots, N) .
$$

Hence, by (2.5), we immediately obtain

$$
\begin{aligned}
& |v|_{1+\theta}^{\theta^{\tau}} \leqslant \bar{K}(0) N A_{2} M^{\lambda} \tau^{(1-\theta) / 2}+\bar{K}(\theta) N A_{3} M \tau^{(1-\theta) / 2}+ \\
& +\left[\vec{K}(\theta) \tau^{(1-\theta) / 2}\left(A_{1}+\left|L^{k} \Phi^{k}\right|_{0}^{\theta^{\tau}}\right)+\left|\Phi^{k}\right|_{1+\theta}^{Q^{\tau}}\right] N .
\end{aligned}
$$

Using (2.6) we now select $M$ so that for $\theta=a$ both the first and the third terms on the right-hand side of $(2.10)$ are equal to or less than the expression

$$
\frac{1}{2}\left[1-\bar{K}(a) N A_{3} \tau^{(1-a) / 2}\right] M
$$

Thus, by (2.10), we have $|v|_{1+a}^{Q^{\tau}} \leqslant M$, i.e. $Z$ maps $C_{M}$ into itself.

It also follows from (2.10) that the set $\left\{Z u: u \in C_{M}\right\}$ is bounded in the space $C_{1+\beta}^{N}\left(G^{\tau}\right)$, whence, by Theorem 1 of [4] (p. 188), this set is a precompact subset of $C_{1+a}^{N}\left(G^{\tau}\right)$.

Note further that $Z$ is continuous, i.e. $\left|u_{l}-u\right|_{1+a}^{Q^{\tau}} \underset{l \rightarrow \infty}{\longrightarrow} 0$ implies $\left|Z u_{l}-Z u\right|_{1+a}^{\sigma^{\tau}} \underset{l \rightarrow \infty}{\longrightarrow} \infty$. Indeed, by the definition of $Z$, we have $v=Z u$ and $v_{l}=Z u_{l}$, where

$$
\begin{gathered}
L^{k} v^{k}=F^{k}(x, t), \quad L^{k} v_{l}^{k}=B^{k} u_{l} \equiv F_{l}^{k}(x, t), \quad(x, t) \epsilon \overrightarrow{G^{k}} \backslash \Sigma^{x}, \\
v^{k}=v_{l}^{k}=p^{k} \text { on } \Sigma^{\pi}(k=1, \ldots, N) .
\end{gathered}
$$

Hence

$$
\begin{gathered}
L^{k}\left(v_{l}^{k}-v^{k}\right)=F_{l}^{k}(x, t)-F^{k}(x, t), \quad(x, t) \in \overline{G^{\tau}} \backslash \Sigma^{t} \\
v_{l}^{k}-v^{k}=0 \quad \text { on } \Sigma^{\tau}(k=1, \ldots, N) .
\end{gathered}
$$


Assumption VII and Lemma 2 applied to (2.11), (2.12) yield the relation

$$
\lim _{l \rightarrow \infty}\left|v_{l}^{k}-v^{k}\right|_{1+a}^{G^{\tau}}=0, \quad \text { i.e. } \quad \lim _{l \rightarrow \infty}\left|Z u_{l^{--}} Z u\right|_{1+a}^{G^{\tau}}=0 .
$$

Finally, since $C_{M}$ is a closed convex set of $C_{1+a}^{N}\left(G^{\tau}\right)$, then by Schauder's theorem $Z$ has a fixed point $u$. Observe that $u$ satisfies (2.1), (2.2) and $u \in C_{1+\beta}^{N}\left(G^{\tau}\right) \cap C_{2+e}^{N}\left(G^{\tau}\right)$ for some $0<\varepsilon<1$, which was to be proved.

Now we derive corollaries from Theorem 1 concerning some special cases of operators $B^{k}$. We introduce the following assumptions:

1. Let $\Psi^{k}(x, t ; z(\cdot, t))\left((x, t) \in \overline{G^{x}}, 1 \leqslant k \leqslant N\right)$ be a functional defined, for every $0<\tau \leqslant T$, on a set of all functions $z(x, t)$ regular in $\overline{G^{\tau}}$ such that for any $z, \bar{z} \in C_{1+a}\left(G^{\top}\right)$ we have the inequality

$$
\left|\Psi^{k}(x, t ; z(\cdot, t))-\Psi^{k}(x, t ; \bar{z}(\cdot, t))\right|_{0}^{G^{\tau}} \leqslant M_{1}|z-\bar{z}|_{0}^{G^{\tau}}
$$

$M_{1}>0$ being a certain constant. Besides, for any $z \in C_{1+a}\left(G^{\tau}\right)$, functions $g^{k}(x, t)=\Psi^{k}(x, t ; z(\cdot, t))$ satisfy a uniform Hölder condition (with exponent $\alpha^{\prime}$ which may depend on $z$ and $k$ ) in $G^{\tau}$.

2. Let functions $f^{k}(x, t, p, q, r) \quad(k=1, \ldots, N)$, defined on $\bar{G} \times$ $\times E_{N+n N+N}$, satisfy a uniform Hölder condition in every bounded set $\bar{G} \times \boldsymbol{H}\left(\boldsymbol{H} \subset E_{N+n N+N}\right)$ and

$$
f^{k}\left(x, 0, \varphi, \varphi_{x}, \Psi(x, 0 ; \varphi(\cdot, 0))\right)=L^{k} \varphi^{k} \quad \text { on } \partial R_{0},
$$

where

$$
\begin{aligned}
u_{x} & =\left(u_{i_{1}}^{1}, \ldots, u_{x_{n}}^{1}, \ldots, u_{x_{1}}^{N}, \ldots, u_{x_{n}}^{N}\right) \\
\Psi(x, t ; u(\cdot, t)) & =\left(\Psi^{1}\left(x, t ; u^{1}(\cdot, t)\right), \ldots, \Psi^{N}\left(x, t ; u^{N}(\cdot, t)\right)\right) .
\end{aligned}
$$

Moreover, there exist constants $N_{1}, N_{2}, N_{3} \geqslant 0,0 \leqslant \lambda<1$, such that

$$
\left|f^{k}(x, t, p, q, r)\right| \leqslant N_{1}+N_{2}|(p, q, r)|^{\lambda}+N_{3}|(p, q, r)|,
$$

where

$$
|(p, q, r)|=\sum_{i=1}^{N}\left|p^{i}\right|+\sum_{i=1}^{N} \sum_{j=1}^{n}\left|q^{i j}\right|+\sum_{i=1}^{N}\left|r^{\cdot i}\right| .
$$

CoRolisary 1. If assumptions I-IV, 1, 2 are fulfilled and

$$
\bar{K}(a) N N_{3}\left(M_{1}+1\right) \tau^{(1-a) / 2}<1,
$$

then Theorem 1 holds true in the case

$$
B^{k} u=f^{k}\left(x, t, u, u_{x}, \Psi(x, l ; u(\cdot, t))\right)\left({ }^{4}\right) .
$$

(4) Expressions of this form occur in differential inequalities of parabolic type treated by Szarski in [10]. 
In order to prove this corollary it suffices to observe that conditions (1), (2), (2.14) imply assumptions V-VII, (2.6) respectively, and to apply Theorem 1.

Corollary 1'. Let assumptions I-IV, 2 and (2.14) be fulfilled and let the measures $\mu^{k}(x, t ; D)$ satisfy all the conditions imposed in section 1 . Then Theorem 1 is true in the case

$$
B^{k} u=f^{k}\left(x, t, u, u_{x}, \int_{G_{t}} u(y, t) \mu(x, t ; d y)\right),
$$

where.

$$
\int_{\sigma_{t}} u(y, t) \mu(x, t ; d y)=\left(\int_{G_{t}} u^{1}(y, t) \mu^{1}(x, t ; d y), \ldots, \int_{G_{t}} u^{N}(y, t) \mu^{N}(x, t ; d y)\right) .
$$

Proof of this corollary follows immediately by applying Lemma 4 and Corollary 1.

At present, under stronger assumptions than those of Theorem 1, we shall prove the existence and uniqueness of solutions of the problem (2.1), (2.2) and the convergence of successive approximations. We retain assumptions I-V whereas VI-VII are replaced by the following ones:

$\mathrm{VI}^{\prime}$. Operators $B^{k}(k=1, \ldots, N)$ map the space $C_{1+a}^{N}\left(G^{z}\right)$ into $C_{a}\left(G^{\tau}\right)$. we have

VII'. There exists a constant $A_{1}^{\prime} \geqslant 0$ such that for any $u, \bar{u} \in C_{1+a}^{N}\left(G^{*}\right)$

$$
\left|B^{k} u-B^{k} \bar{u}\right|_{0}^{G^{x}} \leqslant A_{\mathbf{1}}^{\prime}|u-\bar{u}|_{1}^{G^{\tau}}
$$

THEOREM 2. If assumptions $\mathrm{I}-\mathrm{V}\left({ }^{5}\right), \mathrm{VI}^{\prime}-\mathrm{VII}^{\prime}$ are satisfied and

$$
\bar{K}(a) N A_{1}^{\prime} \tau^{(1-a) / 2}<1,
$$

then problem (2.1), (2.2) has a unique solution $u=\left\{u^{k}\right\}$ in the space $C_{1+a}^{N}\left(G^{\tau}\right)$. Moreover, $u \in C_{1+\beta}^{N}\left(G^{\tau}\right) \cap C_{2+a}^{N}\left(G^{\tau}\right)$.

Proof. Denote by $\Lambda$ the set of all functions $u(x, t) \in C_{1+a}^{N}\left(G^{z}\right)$ such that $u(x, t)=\varphi(x, t)$ on $\Sigma^{r}$. In view of Lemma 1 problem (2.7), (2.8) has, for $u \in A$, a unique solution $v=\left\{v^{k}\right\}$ in the class $C_{2+a}^{N}\left(G^{\tau}\right)$. Moreover, by $(2.5), v \in C_{1+\beta}^{N}\left(G^{\tau}\right)$.

Now we define on $A$ a transformation $Z$ setting $v=Z u$. We shall prove that $Z$ is a contraction in $C_{1+a}^{N}\left(G^{\tau}\right)$. Indeed, let $\bar{v}=Z \bar{u}$. Then $L^{k}\left(v^{k}-\overline{v^{k}}\right)=F^{k}(x, t)-\overline{F^{k}}(x, t)$ for $(x, t) \epsilon \overline{G^{x}} \backslash \Sigma^{x}, v^{k}-\overline{v^{k}}=0$ on $\Sigma^{x}$. Setting $\Phi^{k} \equiv 0$ in (2.5) and making use of assumption VII' we obtain

$$
\left|v^{k}-\bar{v}^{\bar{k}}\right|_{1+a}^{\sigma^{\tau}} \leqslant \bar{K}(\alpha) A_{1}^{\prime} \tau^{(1-\alpha) / 2}|u-\bar{u}|_{1+a}^{Q^{\tau}} .
$$

(8) The condition $a<\beta<1$ may be replaced by $a \leqslant \beta<1$. 
Thus

$$
|Z u-Z \bar{u}|_{1+a}^{\sigma^{\tau}} \leqslant \bar{K}(\alpha) N A_{1}^{\prime} \tau^{(1-\alpha) / q}|u-\bar{u}|_{1+\alpha}^{\sigma^{\tau}},
$$

which means, by (2.17), that $Z$ is a contraction. Since $\Lambda$ is the closed set of $C_{1+a}^{N}\left(G^{\tau}\right)$ and $Z$ maps $\Lambda$ into itself, then by the Banach fixed point theorem $Z$ has a unique fixed point $u$. At the same time we have proved that $u \in C_{1+\beta}^{N}\left(G^{\tau}\right) \cap C_{2+a}^{N}\left(G^{\tau}\right)$. Obviously the function $u$ is a (unique) solution of problem (2.1), (2.2), and the proof is completed.

The solution of the above problem can be obtained by the method of successive approximations. For this purpose we construct a sequence $\left\{u_{l}\right\}=\left\{u_{l}^{1}, \ldots, u_{l}^{N}\right\}$ putting

$$
u_{0}=\Phi, \quad u_{l+1}=Z u_{l} \quad(l=0,1,2, \ldots) .
$$

It follows from the proof of the Banach theorem (see e.g. [4], p. 59) that the sequence $\left\{u_{l}\right\}$ is convergent in $C_{1+a}^{N}\left(G^{\tau}\right)$ to a unique fixed point $u$ of the transformation $Z$. Thus we have relations

$$
\lim _{l \rightarrow \infty} u_{l}^{k}=u^{k} \quad \text { and } \quad \lim _{l \rightarrow \infty}\left(u_{l}^{k}\right)_{x_{l}}=u_{x_{i}}^{k} \quad(k=1, \ldots, N ; i=1, \ldots, n)
$$

where the convergence is uniform. Suppose additionally that

VI'. Operators $B^{k}$ map every bounded subset of $C_{1+a}^{N}\left(G^{\tau}\right)(0<\tau \leqslant T)$ into a bounded subset of $C_{a}\left(G^{\tau}\right)$.

Then, by Lemma 1, the sequence $\left\{\left|u_{l}^{k}\right|_{2+a}^{\sigma^{\tau}}\right\}$ is bounded as well. Hence, in view of Arzela's theorem, there is a subsequence $\left\{u_{l^{\prime}}^{k}\right\}$ such that

$$
\lim _{l^{\prime} \rightarrow \infty}\left(u_{l^{\prime}}^{k}\right)_{x_{i} x_{j}}=u_{x_{l} x_{j}}^{k} \quad \text { and } \quad \lim _{l^{\prime} \rightarrow \infty}\left(u_{l^{\prime}}^{k}\right)_{l}=u_{l}^{k}
$$

where the convergence is uniform.

In order to derive corollaries from Theorem 2 for cases (2.15) and (2.16) we make the following assumptions, instead of assumptions 1, 2:

$1^{\prime}$. Assumption 1 with $\alpha^{\prime}=\alpha$.

$2^{\prime}$. Functions $f^{k}(x, t, p, q, r)$ satisfy a uniform Hölder condition with exponent $a$ in $(x, t) \epsilon \vec{G}$, uniformly with respect to $(p, q, r) \in E_{N+n N+N}$ and condition $(2.13)$ holds true. Furthermore, there is a constant $N_{1}^{\prime} \geqslant 0$ such that

$$
\left|f^{k}(x, t, p, q, r)-f^{k}(x, t, \bar{p}, \bar{q}, \bar{r})\right| \leqslant N_{1}^{\prime}|(p-\bar{p}, q-\bar{q}, r-\bar{r})| .
$$

Corollary 2. If assumptions I-IV, 1', 2' are satisfied and

$$
\bar{K}(a) N N_{1}^{\prime}\left(M_{1}+1\right) \tau^{(1-a) / 2}<1,
$$

then Theorem 2 remains true in case (2.15). 
Corollaky 2'. Let assumptions I-IV, 2' and (2.21) be fulfilled and let the measures $\mu^{k}(x, t ; D)$ satisfy all the conditions imposed in section 1 with $\gamma=a$. Then Theorem 2 is true in case (2.16).

Since assumption $\mathrm{VI}^{\prime \prime}$ is fulfilled in cases (2.15) and (2.16), there exists a subsequence $\left\{u_{l^{\prime}}\right\}$ of sequence $\left\{u_{l}\right\}$ defined by (2.18) such that (2.19) holds.

\section{Lemmas for the parabolic case in an unbounded zone.} Now let $G$ be an unbounded zone $E_{n} \times(0, T)$. We preserve the definition of norms $|u|_{l+a}^{g}(l=0,1,2)$ introduced in section 1 .

Consider the Cauchy problem

$$
\begin{gathered}
L u \equiv \sum_{i, j=1}^{n} a_{i j}(x, t) u_{x x_{j}}+\sum_{i=1}^{n} b_{i}(x, t) u_{x_{i}}+c(x, t) u-u_{t}=f(x, t), \\
(x, t) \in G^{\prime}=E_{n} \times\left(0, T^{\prime}\right], \\
u(x, 0)=\varphi(x), \quad x \in E_{n} .
\end{gathered}
$$

We make the following assumptions:

(A) The operator $L$ is uniformly parabolic in $G^{\prime}$ (see [4], p. 3), whereas its coefficients are bounded and uniformly Hölder continuous, with exponent $a$, in $G^{\prime}$.

(B) The function $\varphi$ together with its first and second order derivatives are bounded in $E_{n}$. Moreover, $\varphi$ and $\varphi_{x_{t}}$ are uniformly Hölder continuous with exponent $\boldsymbol{a}$ in $\boldsymbol{E}_{n}$, while derivatives $\varphi_{x_{i} x_{j}}$ are locally Hölder continuous with exponent $\alpha$ in $E_{n}$.

(C) The function $f(x, t)$ is bounded in $G^{\prime}$ and satisfies a uniform Hölder condition with exponent $\alpha$ in every bounded domain $H \times(0, T]$ $\left(\boldsymbol{H} \subset E_{n}\right)$.

LEMMA 5. If assumptions (A), (B) and (C) are satisfied, then problem (3.1), (3.2) has a unique regular solution $u(x, t)$. Moreover, $u$ and all its derivatives appearing in Lu satisfy a uniform Hölder condition with exponent a in every bounded domain $H \times[\sigma, T]\left(H \subset E_{n}, 0<\sigma<T\right)$.

Proof. The existence and uniqueness are immediate consequences of Theorem 12 of [4] (p. 25) and of Theorem 16 of [4] (p. 29) respectively, whereas the Hölder continuity of $u$ and its derivatives follows from the proof of Theorem 10 of [4] (p. 72).

LEMMA 6. If assumptions (A), (B) and (C) are satisfied, then any regular solution $u(x, t)$ of problem (3.1), (3.2) belongs to $C_{1+a}(G)$. Moreover, there exists a constant $K$ depending only on $a, n, T$ and $L$ such that

$$
|u|_{1+a}^{G^{\tau}} \leqslant K \tau^{(1-a) / 2}\left(|f|_{0}^{G^{\tau}}+|L \varphi|_{0}^{G^{\tau}}\right)+|\varphi|_{1+a}^{E_{n}},
$$

where $G^{\tau}=E_{n} \times(0, \tau), 0<\tau \leqslant T$. 
Proof. Proceeding as in the proof of Lemma 2 of [4] (p. 193) one can derive for a solution $v(x, t)$ of problem $L v=f(x, t)-L \varphi(x)$ for $(x, t) \in G^{\prime}$, $v(x, 0)=0$ in $E_{n}$ the following estimate

$$
|v|_{1+\alpha}^{\left(\sigma^{\tau}\right.} \leqslant K \tau^{(1-\alpha) / 2}|f-L \varphi|_{0}^{\left(\sigma^{\tau}\right.}
$$

which easily implies (3.3).

\section{Existence and uniqueness theorem for parabolic} equations in an unbounded zone. In this section we prove the existence and uniqueness of solutions of the Cauchy problem

$$
\begin{gathered}
L^{k} u^{k}=B^{k} u\left(^{(}\right), \quad(x, t) \in G^{\prime \tau}=E_{n} \times(0, \tau] . \\
u^{k}(x, 0)=\varphi^{k}(x), \quad x \in E_{n}(k=1, \ldots, N) .
\end{gathered}
$$

We make the following assumptions:

I. Operators $L^{k}$ and functions $\varphi^{k}(k=1, \ldots, N)$ satisfy conditions (A) and $(B)$ of the previous section.

II. For every $0<\tau \leqslant T$ and for any $u \in C_{1+a}^{N}\left(G^{\tau}\right)$ functions $B^{k} u$ are bounded in $G^{\prime \tau}$ and satisfy a uniform Hölder condition with exponent $a$ in every bounded domain $H \times(0, \tau]\left(H \subset E_{n}\right)$.

III. Assumption VII' of section 2.

Let functions $F^{k}(x, t)(k=1, \ldots, N)$ satisfy assumption (C) of section 3. Then it follows from assumption $I$ and from Lemma 6 that there exists a constant $K$ depending only on $\alpha, n, T$ and operators $L^{k}$ (considered for $\left.(x, t) \epsilon G^{\prime}\right)$ such that for any regular solution $u^{k}(x, t)(k=1, \ldots, N)$ of the problem $L^{k} u^{k}=F^{k}$ in $G^{\prime \tau}, u^{k}(x, 0)=\varphi^{k}(x)$ in $E_{n}$ we have

$$
\left|u^{k}\right|_{1+a}^{\sigma^{z}} \leqslant K \tau^{(1-\alpha) / 2}\left(\left|F^{k}\right|_{0}^{G^{\tau}}+\left|L^{k} \varphi^{k}\right|_{0}^{G^{x}}\right)+\left|\varphi^{k}\right|_{1+a}^{E_{n}}
$$

THEOREM 3. If assumptions I-III are fulfilled and

$$
N K A_{1}^{\prime} \tau^{(1-a) / 2}<1,
$$

then problem (4.1), (4.2) has a unique regular solution $u(x, t)=\left\{u^{k}(x, t)\right\}$ in class $C_{1+a}^{N}\left(G^{\tau}\right)$. Moreover, derivatives $u_{x_{1},}^{k}$ and $u_{t}^{k}$ are uniformly Hölder continuous of exponent $a$ in every bounded domain $H \times[\sigma, \tau]\left(H \subset E_{n}\right.$, $0<\sigma<\tau)$.

Proof. The proof is similar to that of Theorem 2. Namely, let us denote by $\Lambda$ the set of all functions $u(x, t) \in C_{1+a}^{N}\left(G^{\tau}\right) \operatorname{such}$ that $u(x, 1)$ $=\varphi(x)$ in $E_{n}$ and consider, for $u \in A$, the problem

$$
\begin{aligned}
& L^{k} v^{k}=B^{k} u, \quad(x, t) \in G^{\prime \tau}, \quad(k=1, \ldots, N) . \\
& v^{k}(x, 0)=\varphi^{k}(x), \quad x \in E_{n},
\end{aligned}
$$

(ब) We retain the notation of section 2 . 
By Lemmas 5 and 6 the above problem has a unique regular solution $v^{k}(x, t)(k=1, \ldots, N)$, where $v^{k} \in C_{1+a}\left(G^{\tau}\right)$, whereas $v_{x_{b} x_{j}}^{k}$ and $v_{t}^{k}$ are uniformly Hölder continuous of exponent $\alpha$ in every bounded domain $H \times[\sigma, \tau](0<\sigma<\tau)$. This enables us to define on the set $\Lambda$ a transformation $Z$ by formula $v=Z u$.

Let $\bar{v}=Z \bar{u}$. Then we have

$$
\begin{aligned}
& L^{k}\left(v^{k}-\bar{v}^{k}\right)=B^{k} u-B^{k} \bar{u}, \quad(x, t) \in G^{\prime \tau}, \quad(k=1, \ldots, N) \\
& v^{k}(x, 0)-\bar{v}^{k}(x, 0)=0, \quad x \in E_{n},
\end{aligned}
$$

and hence, by (4.3) and assumption III,

$$
|Z u-Z \bar{u}|_{1+a}^{G^{\tau}} \leqslant N K A_{1}^{\prime} \tau^{(1-a) / 2}|u-\bar{u}|_{1+a}^{G^{\tau}}
$$

Thus, by (4.4), $Z$ is a contraction. Since the remaining assumptions of the Banach theorem are also fulfilled, $Z$ has a unique fixed point which completes the proof.

The solution of problem (4.1), (4.2) can be obtained as a limit of the sequence of successive approximations defined similarly as in section 2.

Now we formulate Theorem 3 for cases (2.15) and (2.16). For this purpose instead of assumption $2^{\prime}$ of section 2 we introduce the following one:

2". Functions $f^{k}(x, t, p, q, r)(k=1, \ldots, N)$ are uniformly Hölder continuous with exponent $a$ in $(x, t)$ in every bounded domain $H \times(0, T]$ uniformly with respect to $(p, q, r) \in E_{N+n N+N}$. Moreover, functions $f^{k}(x, t, 0,0,0)(k=1, \ldots, N)$ are bounded in $G^{\prime}$ and condition $(2.20)$ is fulfilled.

CoRollary 3. If assumptions I, $2^{\prime \prime}$ and $1^{\prime}$ of section 2 are satisfied and

$$
N K N_{1}^{\prime}\left(M_{1}+1\right) t^{(1-a) / 2}<1
$$

then Theorem 3 remains true for case (2.15).

Corollary $3^{\prime}$. Let assumptions I, 2", (4.5) be satisfied and let the measures $\mu^{k}(x, t ; D)$ fulfil conditions (1) and (2) (with $\gamma=\alpha$ ) of section 1. Under these assumptions Theorem 3 is true for case (2.16).

5. Lemmas for the ellptic case. The elliptic problem will only be treated in a bounded domain. So let $G$ be an open bounded domain of the Euclidean space $E_{n}$ of the variables $x=\left(x_{1}, \ldots, x_{n}\right)$. Following Friedman [4] we formulate several definitions concerning norms, sets of functions, properties of the boundary $\partial G$ of domain $G$ and functions defined on $\partial G$. 
The following norms are introduced:

$$
\begin{gathered}
|u|_{0}^{G}=\sup _{x \in G^{T}}|u(x)|, \quad|u|_{a}^{G i}=|u|_{0}^{G}+\sup _{x, x^{\prime} \in G} \frac{\left|u(x)-u\left(x^{\prime}\right)\right|}{\left|x-x^{\prime}\right|^{a}}, \\
|u|_{1+a}^{G}=|u|_{0}^{G}+\sum_{i=1}^{n}\left|u_{x_{i}}\right|_{a}^{G}, \quad|u|_{2+a}^{G}=|u|_{0}^{G}+\sum_{i=1}^{n}\left|u_{x_{1}}\right|_{0}^{\sigma}+\sum_{i, j=1}^{n}\left|u_{x_{i} x}\right|_{a}^{a} \\
(0<a<1) .
\end{gathered}
$$

The set of all functions $u$ with finite norm $|u|_{l+a}^{G}(l=0,1,2)$ will be denoted by $C_{l+a}(G)$.

For every point $x \in \partial G$ let there exist an $n$-dimensional neighbourhood $V$ such that $V \cap \partial G$ can be represented, for some $i(1 \leqslant i \leqslant n)$, by an equation of the form

$$
x_{i}=h\left(x_{1}, \ldots, x_{i-1}, x_{i+1}, \ldots, x_{n}\right) .
$$

If the functions $h$ belong to $C_{2+a}$, then we say that $\partial G$ is of class $C_{2+a}$.

A function $\varphi$ defined on $\partial G$ is said to be of class $C_{2+a}$ if it has an extension $\Phi \in C_{2+a}(G)$. Then we define

$$
|\varphi|_{2+a}^{a}=\inf _{\Phi}|\Phi|_{2+a}^{G}
$$

Consider the Dirichlet problem:

$$
\begin{gathered}
L u=\sum_{i, j=1}^{n} a_{i j}(x) u_{s_{i} x_{j}}+\sum_{i=1}^{n} b_{i}(x) u_{x_{i}}+c(x) u=f(x), \quad x \in G, \\
u(x)=\varphi(x), \quad x \in \partial G .
\end{gathered}
$$

We shall need the following assumptions:

(A) For any $x \in G$ and $\xi \in E_{n}$ we have

$a_{i j}(x)=a_{j i}(x), \quad c(x) \leqslant 0, \quad \sum_{i, j=1}^{n} a_{i ;}(x) \xi_{i} \xi_{j} \geqslant K_{0}|\xi|^{2} \quad\left(K_{0}=\right.$ const $\left.>0\right)$.

(B) The coefficients of $L$ and the function $f$ are uniformly Hölder continuous with exponent $\alpha$ in $G$. Thus for some constant $K_{1} \geqslant 0$

$$
\left|a_{i j}\right|_{a}^{\alpha}, \quad\left|b_{i}\right|_{a}^{\theta}, \quad|c|_{a}^{G} \leqslant K_{1} .
$$

From [4], p. 86, and [3], p. 808 (see also [1]) we obtain the following

LEMma 7. If assumptions (A) and (B) hold true, the boundary $\partial G$ is of class $C_{2+a^{\prime}}\left(a<a^{\prime}<1\right)$ and $\varphi \in C_{2+a}$, then problem (5.1), (5.2) has a unique regular $\left({ }^{7}\right)$ solution $u(x)$ in the class $C_{2+a}(G)$. Moreover, there

(7) I.e. continuous in $\bar{G}$ and possessing continuous first and second order derivatives in $G$. 
exist constants $K, \beta$ (depending only on $L$ and $G$ ) and $K^{\prime}$ (depending only on $K_{0}, K_{1}, a$ and $G$ ) such that

$$
|u|_{1+\beta}^{G} \leqslant K\left(|f|_{0}^{G}+\mid L \Phi_{\mid 0}^{G}\right)+|\Phi|_{1+\beta}^{G}\left(^{(8)} .\right.
$$

and

$$
|u|_{2+\alpha}^{G} \leqslant K^{\prime}\left(|\varphi|_{2+a}^{G}+|f|_{a}^{G}\right),
$$

where $\Phi \in C_{2+a}(G)$ is any extension of $\varphi$.

Now denote by $\mathcal{A}$ the $\sigma$-field of all Lebesgue-measurable subscts of the closure $\vec{G}$. Let $\mu(x ; D)$ be a non-negative measure defined on $\mathcal{M}$, depending on $x \in \bar{G}$, and satisfying the following conditions:

(1) there is a constant $M_{1}>0$ such that

for any $x \in \bar{G}$;

$$
\mu(x ; \bar{G}) \leqslant M_{1}
$$

(2) there exists a finite non-negative measure $\bar{\mu}$ defined on $\mathcal{N}$ such that for any $D \in \mathcal{N}$ and $x, \dot{x}^{\prime} \in \bar{G}$

$$
\left|\mu(x ; D)-\mu\left(x^{\prime} ; D\right)\right| \leqslant \bar{\mu}(D)\left|x-x^{\prime}\right|^{\nu},
$$

where $0<\gamma<1$ is a constant.

By an argument similar to that used in the proof of Lemma 4, one can prove the following

LEMMA 8. If a function $u(x)$ is uniformly Hölder continuous with exponent $a$ in $G$, then the function

$$
v(x)=\int_{G} u(y) \mu(x ; d y)
$$

is uniformly Hölder continuous with the exponent $\bar{\alpha}=\min (\alpha, \gamma)$ in the domain $G$.

6. Existence theorems for elliptic equations. In the present section we discuss the existenee of solutions of the Dirichlet problem

$$
\begin{gathered}
L^{k} u^{k} \equiv \sum_{i, f=1}^{n} a_{i j}^{k}(x) u_{x_{i x}}^{k}+\sum_{i=1}^{n} b_{i}^{k}(x) u_{x_{i}}^{k}+c^{k}(x) u^{k}=B^{k} u, \quad x \in G, \\
u^{k}(x)=\varphi^{k}(x), \quad x \in \partial G(k=1, \ldots, N),
\end{gathered}
$$

where $B^{k}$ is an operator defined on the set of all vector-functions $u=\left\{u^{k}\right\}$ regular in $\bar{G}$ with values belonging to the set of all functions defined in $G$.

For $i, j=1, \ldots, n ; k=1, \ldots, N$ we need the following assumptions:

( $\left.{ }^{8}\right)$ It is easy to see that relation $\Phi \in C_{2+\alpha}(G)$ implies $\Phi \in C_{1+\beta}(G)$ for any $0<\beta<1$. Annales Polonicl Mathematicl XXII 
I. For any $x \in G$ and $\xi \in E_{n}$ we have

$a_{i j}^{k}(x)=a_{j i}^{k}(x), \quad c^{k}(x) \leqslant 0, \quad \sum_{i, j=1}^{n} a_{i j}^{k}(x) \xi_{i} \xi_{j} \geqslant K_{0}|\xi|^{2} \quad\left(K_{0}=\right.$ const $\left.>0\right)$.

II. The coefficients of $L^{k}$ are uniformly Hölder continuous with exponent $a$ in $G$. Hence, for some constant $K_{1}>0$, we have

$$
\left|a_{i j}^{k}\right|_{a}^{G}, \quad\left|b_{i}^{k}\right|_{a}^{G}, \quad\left|c^{k}\right|_{a}^{\theta} \leqslant K_{1} .
$$

III. The boundary $\partial G$ is of class $C_{2+a^{\prime}}\left(\alpha<a^{\prime}<1\right)$ and $p^{k} \in C_{2+a}$. In order to formulate further assumptions we introduce some symbols. Assume that functions $F^{k}(x)(k=1, \ldots, N)$ are uniformly Hölder continuous with exponent $a$ in $G$. Then, by assumptions I-III and by Lemma 7, the problem

$$
L^{k} v^{k}=F^{k} \quad \text { in } G, v^{k}=\varphi^{k} \text { on } \partial G(k=1, \ldots, N)
$$

has a unique solution $v^{k}(x)$. Moreover, there exist constants $K$ and $\beta$ (depending only on $L^{k}$ and domain $G$ ) such that

$$
\left|v^{k}\right|_{1+\beta}^{G} \leqslant K\left(\left|F^{k}\right|_{0}^{a}+\left|L^{k} \Phi^{k}\right|_{0}^{G}\right)+\left|\Phi^{k_{1+\beta}}\right|_{1+\beta},
$$

where $\Phi^{k} \in C_{2+a}(G)$ is any extension of $\varphi^{k}$. Now let $A_{1}$ and $\beta^{\prime}$ be such constants that

$$
\begin{gathered}
0 \leqslant A_{1}<(K N)^{-1}, \\
R^{\beta-\beta^{\prime}} \leqslant 1+\Lambda / 2, \quad 0<\beta^{\prime}<\beta,
\end{gathered}
$$

where $R$ is the diameter of the domain $G$ and $\Delta=1-K N A_{1}$.

IV. Operators $B^{k}$ map the space $C_{1+\beta^{\prime}}^{N}(G)\left(^{\theta}\right)$ into the set $\bigcup_{0<\theta<1} C_{\theta}(G)$ and there exist constants $A_{2}, A_{3} \geqslant 0,0 \leqslant \lambda<1$ such that for any $u \in C_{1+\beta^{\prime}}^{N}(G)$ we have the inequality

$$
\left|B^{k} u\right|_{0}^{G} \leqslant A_{1}|u|_{1}^{G}+A_{2}\left(|u|_{1}^{G}\right)^{\lambda}+A_{3} .
$$

V. Operators $B^{k}$ are continuous in the space $C_{1+\beta^{\prime}}^{N}(G)$, i.e. if $u, u_{l} \epsilon$ $\epsilon C_{1+\beta^{\prime}}^{N}(G)$ and $\lim _{l \rightarrow \infty}\left|u_{l}-u\right|_{1+\beta^{\prime}}^{\sigma}=0$, then $\lim _{l \rightarrow \infty}\left|B^{k} u_{l}-B^{k} u\right|_{0}^{G}=0$.

Theorem 4. If assumptions I-V are satisfied, then problem (6.1), (6.2) has a solution $u=\left\{u^{k}\right\}$, which belongs to $C_{2+\theta}^{N}(G)$ for some $0<\varepsilon<1$.

Proof. Denote by $C_{M}$ the set of all functions $u(x) \in C_{1+\beta^{\prime}}^{N}(G)$ such that $|u|_{1+\beta^{\prime}}^{G} \leqslant M$ and $u(x)=\varphi(x)$ on $\partial G$, where $M>0$ is a constant to be conveniently chosen.

( $)$ We retain, with obvious modifications, the notation introduced in section 2 . 
Consider now, for $u \in C_{M}$, the problem

$$
\begin{aligned}
& L^{k} v^{k}=B^{k} u \equiv F^{k}(x), \quad x \in G, \quad v^{k}(x)=\varphi^{k}(x), \quad x \in \partial G \\
& (k=1, \ldots, N) \text {. }
\end{aligned}
$$

This problem, by Lemma 7, has a unique solution $v=\left\{v^{k}\right\}$ belonging to the class $C_{2+\varepsilon}^{N}(G)$ for some $0<\varepsilon<1$. Now we define a transformation $Z$ setting $v=Z u$. Applying (6.3) we obtain

$$
\left|v^{k}\right|_{1+\beta^{\prime}}^{G} \leqslant \max \left(1, R^{\beta-\beta^{\prime}}\right)\left[K\left(\left|F^{k}\right|_{0}^{\boldsymbol{G}}+\left|L^{k} \Phi^{k}\right|_{0}^{\boldsymbol{G}}\right)+\left|\Phi^{k}\right|_{1+\beta}^{G}\right] .
$$

Hence, by (6.6) and (6.5),

$$
\begin{aligned}
|v|_{1+\beta^{\prime}}^{a} \leqslant(1+\Delta / 2) N\left[K\left(A_{3}+\left|L^{k} \Phi^{k}\right|_{0}^{a}\right)+\left|\Phi^{k}\right|_{1+\beta}^{G}\right]+ & \\
& +(1+\Delta / 2) K A_{2} N M^{\lambda}+(1+\Delta / 2) K A_{1} N M .
\end{aligned}
$$

Choosing the constant $M$ so that the sum of the first and the second term on the rigth-hand side of the last inequality is less than or equal to $\frac{\Delta^{2}+\Delta}{2} M$, we get, by the definition of $\Delta,|v|_{1+\beta^{\prime}}^{G} \leqslant M$. Thus $Z$ maps $C_{M}$ into itself. The further argumentation is the same as in the proof of Theorem 1.

As in section 2, we shall consider now some special cases of operators $B^{k}$ for which Theorem 4 holds true. We introduce the following assumptions:

1. Let $\Psi^{k}(x ; z(\cdot))(x \in G, k=1, \ldots, N)$ be a functional defined on the set of all functions $z(x)$ regular in $\bar{G}$ such that for any $z, \bar{z} \epsilon C_{1+\beta^{\prime}}(G)$ we have the inequality

$$
\left|\Psi^{k}(x ; z(\cdot))-\Psi^{k}(x ; \bar{z}(\cdot))\right|_{0}^{G} \leqslant M_{1}|z-\bar{z}|_{0}^{G},
$$

$M_{1}>0$ being a certain constant. Besides, for any $z \in C_{1+\beta^{\prime}}(G)$ the function $g^{k}(x)=\Psi^{k}(x ; z(\cdot))$ satisfies a uniform Hölder condition (with exponent $\bar{\beta}$ which may depend on $z$ and $k$ ) in $G$.

2. Let functions $f^{k}(x, p, q, r)(k=1, \ldots, N)$, defined on $G \times E_{N+n N+N}$, satisfy a uniform Hölder condition in every bounded set $G \times H$ $\left(H \subset E_{N+n N+N}\right)$. Moreover, there are constants $N_{1}, N_{2} \geqslant 0$ and $0 \leqslant \lambda<1$ such that

$$
\left|f^{k}(x, p, q, r)\right| \leqslant A_{1}\left(M_{1}+1\right)^{-1}|(p, q, r)|+N_{1}|(p, q, r)|^{\lambda}+N_{2} .
$$

COROLLARY 4. If assumptions I-III and 1, 2 are satisfied, then the assertion of Theorem 4 remains valid with

$$
B^{k} u=f^{k}\left(x, u, u_{x}, \Psi(x ; u(\cdot))\right) .
$$


CoROLLARY 4'. If assumptions I-III, 2 and 1, 2 with $\left(\mu=\mu^{k}\right)$ of section 5 are satisfied, then Theorem 4 is true for operators $B^{k}$ given by the formulas

$$
B^{k} u=f^{k}\left(x, u, u_{x}, \int_{\theta} u(y) \mu(x ; d y)\right) .
$$

Now, under stronger assumptions than those of Theorem 4, we shall prove the existence and uniqueness of solutions of problem (6.1), (6.2) and the convergence of the successive approximations. Instead of assumptions IV, V we make the following ones (assumptions I-III' being retained):

$\mathrm{IV}^{\prime}$. Operators $B^{k}(k=1, \ldots, N)$ map the space $C_{1+\beta}^{N}(G)$ into $C_{\delta}(G)$, where $\delta=\min (\alpha, \beta)$. we have

$\mathrm{V}^{\prime}$. There is a constant $A_{\mathbf{1}}^{\prime} \geqslant 0$ such that for any $u, \bar{u} \in C_{1+\beta}^{N}(G)$

$$
\left|B^{k} u-B^{k} \bar{u}\right|_{0}^{G} \leqslant A_{1}^{\prime}|u-\bar{u}|_{1}^{G} .
$$

THEOREM 5. Let assumptions I-III $\left({ }^{10}\right), \mathrm{IV}^{\prime}, \mathrm{V}^{\prime}$ be fulfilled and let

$$
A_{\mathrm{i}}^{\prime}<(K N)^{-1} \text {. }
$$

Under these assumptions problem (6.1), (6.2) has a unique solution $u=\left\{u^{k}\right\}$ of class $C_{1+\beta}^{N}(G)$. Moreover, $u \in C_{2+\delta}^{N}(G)$.

Proof. The proof is similar to that of Theorem 2. Namely, let us denote by $\Lambda$ the set of all functions $u(x) \in C_{1+\beta}^{N}(G)$, such that $u(x)=\varphi(x)$ on $\partial G$ and consider problem (6.7) for $u \epsilon \Lambda$. Next, using the theorems on the existence and uniqueness of solutions of this problem we define the transformation $v=Z u$. We show that $Z$ maps $\Lambda$ into $\Lambda \cap C_{2+\delta}^{N}(G)$ and that $Z$ is a contraction in $\Lambda$. This enables us to apply the Banach fixed point theorem, from which the proof of the theorem follows.

As in the parabolic case the solution of problem (6.1), (6.2) can be obtained by the method of successive approximations.

To end this section, we formulate Theorem 5 for cases (6.9) and (6.10). For this purpose we make, instead of assumptions 1, 2, the following ones:

$1^{\prime}$. Assumption 1 with $\beta^{\prime}=\beta$ and $\bar{\beta}=\delta$.

$2^{\prime}$. Functions $f^{k}(x, p, q, r)$ are uniformly Hölder continuous with exponent $\delta$ in $x \in G$, uniformly with respect to $(p, q, r) \in E_{N+n N+N}$. Moreover, there is such a constant $N_{\mathbf{i}}^{\prime}>0$ that

$$
\left|f^{k}(x, p, q, r)-f^{k}(x, \bar{p}, \bar{q}, \bar{r})\right| \leqslant N_{1}^{\prime}|(p-\bar{p}, q-\bar{q}, r-\bar{r})| .
$$

Corollary 5. Let assumptions I-III, $1^{\prime}, 2^{\prime}$ be satisfied and let

$$
N_{1}^{\prime}<\left[K N\left(M_{1}+1\right)\right]^{-1} .
$$

(10) In III, instead of assumption $\varphi^{k} \in C_{2+a}$, we may only assume that $\varphi^{k} \in C_{2+8}$. 
Then Theorem 5 holds true in case (6.9).

CoRollary 5'. If assumptions I-III, 2', 1, 2 (with $\mu=\mu^{k}$ and $\gamma=\delta$ ) of section 5 and condition (6.12) are satisfied, then the conclusion of Theorem 5 holds true in the case of the operators given by (6.10).

\section{References}

[1] H. O. Cordes, Über die erste Randwertaufgabe bei quasilinearen Differentialgleichungen zweiter Ordnung in mehr als zwei Variablen, Math. Ann. 131 (1956), pp. 278-312.

[2] С. Д. Зйдельман, Параболические системы, Издательство „Наука”, Москва 1964.

[3] A. Friedman, On quasi-linear parabolic equations of the second order, Journal of Math. and Mech. 7 (1958), pp. 793-809; II, 9 (1960), pp. 539-556.

[4] - Partial differential equations of parabolic type, Prentice-Hall, Englewood Cliffs 1964.

[5] M. Krzyżański, Principe d'extremum relatif aux solutions de l'équation intégrodifférentielle du processus stochastique markovien purement discontinu, Bull. Acad. Polon. Sci. 11 (8) (1963), pp. 531-534.

[6] - Principe d'extremum relatif aux solutions de l'équation intégro-différentielle du processus stochastique markovien mixte, Ann. Polon. Math. 16 (1965), pp. 365-370.

[7] T. Kusano, On the first boundary problem for quasi-linear systems of parabolic differential equations in non-cylindrical domains, Funkcjalaj Ekvacioj (Serio Internacia), volumo 7 (1965), pp. 103-118.

[8] I. Łojczyk-Królikiewicz, Certaines inégalités intégro-différentielles, Bull. Acad. Polon. Sci. 14 (2) (1966), pp. 71-75.

[9] - and J. Szarski, On a non-linear system of parabolic integro-differential inequalities in an unbounded region, Ann. Polon. Math. 19 (1967), pp. 61-67.

[10] J. Szarski, Sur un système non-linéaire d'inégalités différentielles paraboliques contenant des fonctionelles, Coll. Math. 16 (1967), pp. 141-145. 